\title{
Morfologia e morfometria do encéfalo de avestruz (Struthio camelus)
}

Received: Feb, 2020; Accepted: Jun, 2020

\author{
Shirley Viana Peçanha", José Miguel Farias Hernandez ${ }^{2}$, \\ Renata Medeiros do Nascimento ${ }^{1}$, Paulo Souza Junior ${ }^{3}$, \\ Marcelo Abidu Figueiredo ${ }^{1 *}$
}

\begin{abstract}
Resumo: O avestruz africano é uma ave ratita da família Struthionidae. As características anatômicas do encéfalo do avestruz foram investigadas neste artigo. Vinte encéfalos de avestruzes adultos (dez machos e dez fêmeas), fixados em formaldeído a $10 \%$, foram analisados neste estudo. Foram obtidas as medidas do encéfalo: comprimento e largura total do encéfalo, comprimento, largura e altura dos hemisférios cerebrais direito e esquerdo, comprimento e largura do cerebelo, comprimento e largura dos lobos ópticos direito e esquerdo. Também foram aferidos o comprimento e a largura do crânio. Os resultados para cada variável foram expressos em média e em desvio padrão. Através do teste $t$ de Student não-pareado as variáveis foram comparadas entre os sexos e entre os antímeros. Considerou-se $\mathrm{p}<0,05$ como significante. $\mathrm{O}$ comprimento e largura do encéfalo dos machos foram de $5,57 \mathrm{~cm} \pm 0,23 \mathrm{~cm}$ e de $4,19 \mathrm{~cm} \pm 0,16 \mathrm{~cm}$, respectivamente. Nas fêmeas o comprimento e largura do encéfalo foram de $5,56 \mathrm{~cm} \pm 0,23 \mathrm{~cm}$ e de $4,18 \mathrm{~cm} \pm 0,16 \mathrm{~cm}$ respectivamente. $\mathrm{O}$ comprimento do hemisfério cerebral direito foi de $3,41 \mathrm{~cm} \pm 0,13 \mathrm{~cm}$ nos machos e de $3,39 \mathrm{~cm} \pm 0,12 \mathrm{~cm}$ nas fêmeas, já no esquerdo foi de $3,35 \mathrm{~cm} \pm$ $0,14 \mathrm{~cm}$ nos machos e de $3,34 \mathrm{~cm} \pm 0,05 \mathrm{~cm}$ nas fêmeas. A largura do hemisfério cerebral direito foi de $2,27 \mathrm{~cm} \pm 0,17 \mathrm{~cm}$ nos machos e de 2,25 \pm $0,14 \mathrm{~cm}$ nas fêmeas, e do esquerdo foi de $2,25 \mathrm{~cm} \pm 0,11 \mathrm{~cm}$ nos machos e de $2,27 \mathrm{~cm} \pm 0,11 \mathrm{~cm}$ nas fêmeas. A altura do hemisfério cerebral direito foi de $2,08 \mathrm{~cm} \pm 0,23 \mathrm{~cm}$ nos machos e de $2,07 \mathrm{~cm} \pm 0,21 \mathrm{~cm}$ nas fêmeas e do hemisfério cerebral esquerdo foi de $2,05 \mathrm{~cm} \pm 0,22 \mathrm{~cm}$ nos machos e de 2,05 $\mathrm{cm} \pm 0,22$ nas fêmeas. $\mathrm{O}$ comprimento do cerebelo foi de $2,45 \mathrm{~cm} \pm 0,32 \mathrm{~cm}$
\end{abstract}

\footnotetext{
1 Universidade Federal Rural do Rio Janeiro. Instituto de Ciências Biológicas e da Saúde Departamento de Anatomia Animal e Humana. BR-465, Km 7 Seropédica- Rio de Janeiro

2 Universidade Federal Rural do Rio Janeiro. Instituto de Veterinária - Departamento de Epidemiologia e Saúde Pública. BR-465, Km 7 Seropédica- Rio de Janeiro.

${ }^{3}$ Universidade Federal do Pampa, campus Uruguaiana. Laboratório de Anatomia Animal. Rodovia BR-472, Km 595, Uruguaiana-Rio Grande do Sul.
}

*Corresponding author: Marcelo Abidu Figueiredo. Universidade Federal Rural do Rio Janeiro. Instituto de Ciências Biológicas e da Saúde - BR-465, Km 7 Seropédica - Rio de Janeiro CEP. 23.897000 Fone: (21) 2682-1763. E-mail: marceloabidu@gmail.com 
nos machos e de $2,44 \mathrm{~cm} \pm 0,32 \mathrm{~cm}$ nas fêmeas, e sua largura foi de $1,59 \mathrm{~cm}$ $\pm 0,16 \mathrm{~cm}$ nos machos e de $1,58 \mathrm{~cm} \pm 0,15 \mathrm{~cm}$ nas fêmeas. $\mathrm{O}$ comprimento do lobo óptico direito foi de $1,30 \mathrm{~cm} \pm 0,06$ nos machos e de $1,30 \mathrm{~cm} \pm 0,06$ $\mathrm{cm}$ nas fêmeas, já o comprimento do lobo óptico esquerdo foi de $1,24 \mathrm{~cm} \pm$ $0,07 \mathrm{~cm}$ nos machos e de $1,23 \mathrm{~cm} \pm 0,67 \mathrm{~cm}$ nas fêmeas. A largura do lobo óptico direito foi de $1,08 \mathrm{~cm} \pm 0,07 \mathrm{~cm}$ nos machos e de $1,08 \mathrm{~cm} \pm 0,07 \mathrm{~cm}$ nas fêmeas e largura do lobo óptico esquerdo foi de $1,08 \mathrm{~cm} \pm 0,07 \mathrm{~cm}$ nos machos e de $1,08 \mathrm{~cm} \pm 0,07 \mathrm{~cm}$ nas fêmeas. A superfície do cérebro do avestruz é lisa sem qualquer giro ou sulco. A média do comprimento dos crânios dos machos é maior que a das fêmeas. Em relação aos 20 encéfalos analisados o lobo óptico direito é maior que o esquerdo. Houve correlação linear positiva entre a largura do crânio com a largura do cerebelo.

Palavras-chave: Anatomia, Aves, Ratitas, Sistema nervoso.

\section{Introdução}

Existe uma grande variação na forma do cérebro entre as aves. As diferenças nas dimensões do cérebro refletem capacidades sensoriais e repertórios comportamentais específicos da espécie, que são moldados por fatores ambientais e biológicos durante a evolução (KAWABE et al., 2013).

Uma caracterização apropriada da morfologia cerebral é crítica para avaliar diferenças neuroanatômicas entre os táxons e gerar conclusões precisas sobre como e por que essas diferenças evoluíram (STRIEDTER, 2009).

Uma relação geral de desenvolvimento entre cérebro e crânio, com ênfase na região facial, tem sido documentada nos tetrápodes (RICHTSMEIER e FLAHERTY, 2013).

Os principais grupos de vertebrados tetrápodes diferem significativamente um do outro no tamanho relativo do encéfalo (YU, ASHWELL e SHULRUF, 2019). Os encéfalos das aves, por exemplo, são quatro a seis vezes maiores que os encéfalos de répteis com o mesmo tamanho corporal, e os vertebrados endotérmicos em geral têm tamanhos encefálicos, aproximadamente, dez vezes maiores que os vertebrados ectotérmicos do mesmo tamanho corporal (STRIEDTER, 2009; KÖNIG, KOLBEL e LIEBICH, 2016).

A evolução independente do aumento do encéfalo entre mamíferos e aves está refletida em diferentes linhas que se ajustam ao tamanho do 
encéfalo: as aves pequenas tendem a ser mais encefalizadas do que os mamíferos de porte pequeno; mas as aves grandes tendem a ser menos encefalizadas que os mamíferos de grande porte (STRIEDTER, 2009; KÖNIG, KOLBEL e LIEBICH, 2016).

Em vertebrados, o tamanho absoluto do encéfalo geralmente aumenta com o tamanho do corpo (LÜPS, 2010). Em estudos experimentais e comparativos, Kotrschal et al. (2015) mostraram que, em aves, considerar o tamanho do corpo ao medir o tamanho do encéfalo pode fornecer uma indicação de certos aspectos da cognição, como resolução de problemas e inovação comportamental.

O encéfalo é o centro de controle das atividades vitais necessárias para a sobrevivência. Ele recebe impulsos dos órgãos sensoriais através da medula espinhal e dos nervos cranianos, processa esses impulsos e inicia saídas motoras para órgãos efetores. Assim, o cérebro é o órgão especializado em coordenar atividades em relação às mudanças nos ambientes interno e externo (KÖNIG, KOLBEL e LIEBICH, 2016).

O encéfalo adulto é dividido no prosencéfalo (telencéfalo e diencéfalo), no mesencéfalo (mesencéfalo) e no rombencéfalo (metencéfalo e mielencéfalo). O encéfalo pode ser dividido em cérebro, cerebelo e tronco encefálico (PENG et al., 2010).

Atualmente há um interesse em diversas espécies de aves por sua utilização como modelos experimentais em pesquisas biológicas (GEUTHNER et al., 2019), e como fontes de proteína de alta qualidade (ASPRILLA-PEREA e DÍAZ-PUENTE, 2019). Como resultado desse interesse em aves em diferentes níveis, há um aumento na compreensão de sua anatomia e de sua fisiologia.

Embora compartilhe de características anatômicas com outros grupos de aves, o avestruz possui muitas peculiaridades que continuam sem entendimento, como sua morfofuncionalidade, que carecem de descrições 
mais detalhadas; valores morfométricos de encéfalos de avestruzes são escassos na literatura, necessitando de maiores investigações.

A aparência macroscópica da superfície do cérebro difere nos vertebrados, dependendo de suas diferentes adaptações e estilos de vida. Podem ser classificados em girencefálicos possuindo sulcos e giros ou em lisencefálicos, quando a superfície se apresente lisa (KÖNIG, KOLBEL e LIEBICH, 2016).

O objetivo deste trabalho é caracterizar a morfologia e a morfometria do encéfalo de avestruzes adultos, comparando os resultados entre machos e fêmeas fornecendo, dessa maneira, subsídios para a área de anatomia comparada e para a avicultura.

\section{Material e métodos}

Vinte avestruzes adultos com 13 meses de idade (dez machos e dez fêmeas), provenientes de criatórios comerciais na região de Itaboraí (Rio de Janeiro), pesando em média $140 \mathrm{~kg}$, foram utilizados neste estudo. Esse material foi coletado em abatedouro local, o que dispensa a obrigatoriedade da submissão e da aprovação pelo comitê de ética. Essas aves foram insensibilizadas por eletrochoque e submetidas a sangria e, consequentemente, mortas por hipovolemia. Segmentos contendo cabeça e pescoço foram acomodados em caixas isotérmicas de isopor e doados ao Departamento de Anatomia Animal e Humana da Universidade Federal Rural do Rio de Janeiro. No laboratório, as peças cadavéricas foram lavadas em água corrente por 20 minutos e fixadas com solução de formaldeído a $10 \%$ através da introdução de uma sonda plástica na artéria carótida. As cabeças foram acondicionadas em caixas de polietileno de baixa densidade com capacidade de 500 litros, contendo a mesma solução, por sete dias para a finalização do processo de fixação. 
Decorridos os sete dias, as cabeças foram retiradas das caixas e lavadas em água corrente por 20 minutos e, com auxílio de um paquímetro de precisão $(0,05 \mathrm{~mm})$, foi feita a mensuração do comprimento do crânio (distância medida entre a porção rostral do osso frontal e a porção caudal do osso occipital) e da largura do crânio (distância entre os processos pós orbitais) (Figura 1).

Figura 1: Fotomacrografia do crânio de avestruz em vista dorsal. C: comprimento do crânio. L: largura do crânio. Barra de escala: $1 \mathrm{~cm}$

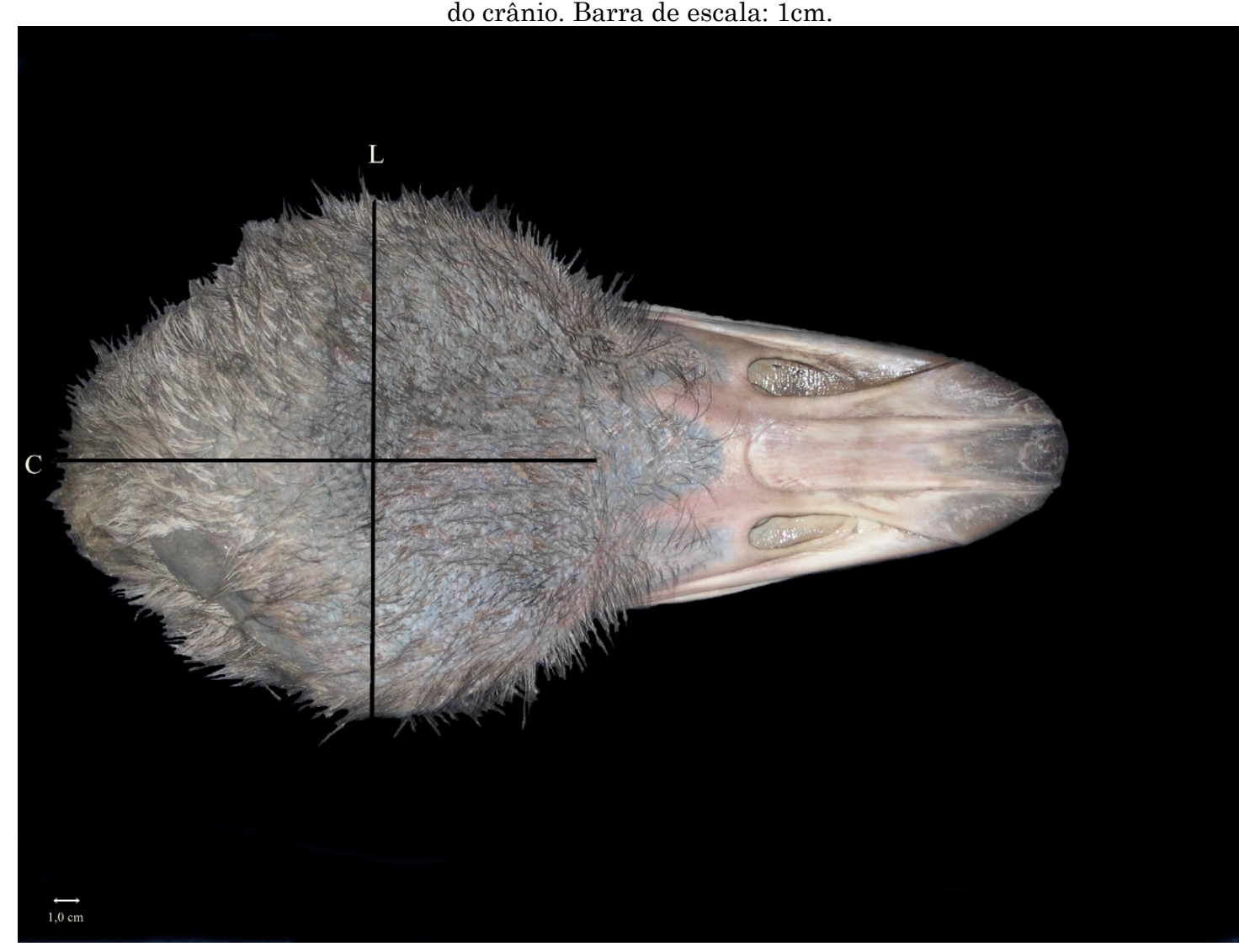

Em seguida, com auxílio de bisturi e de pinça de dissecção, a pele e os tecidos adjacentes foram removidos. Posteriormente, com uso de fórceps odontológico os crânios foram abertos na região do osso frontal e parietal, de modo a possibilitar a remoção do encéfalo. Com o mesmo paquímetro foram obtidas as medidas do encéfalo: comprimento e largura total do encéfalo, comprimento, largura e altura dos hemisférios cerebrais direito e esquerdo, 
comprimento e largura do cerebelo, comprimento e largura dos lobos ópticos direito e esquerdo (PENG et al., 2010). A documentação fotográfica foi realizada usando câmera digital Nikon Coolpix L820.

Os resultados para cada variável foram expressos em média e desvio padrão (Tabelas 1 e 2). Através do teste $t$ de Student não-pareado, as variáveis foram comparadas entre os sexos e entre os antímeros. Foi calculada a correlação linear simples entre o comprimento e a largura do crânio com o comprimento e a largura do encéfalo, hemisfério cerebral direito e esquerdo, cerebelo e lobo óptico direito e esquerdo. Considerou-se como significância estatística o valor de $\mathrm{P}<0,05$. Toda a análise foi realizada no software Graphpad Prism $5^{\circledR}$.

\section{Resultados}

A média do comprimento dos crânios dos machos é maior que o das fêmeas $(\mathrm{P}$-valor $=0,04)($ Tabela 1$)$. Na contagem geral o lobo óptico direito é maior que o esquerdo (P-valor $=0,01)$ (Tabela 2). Houve Correlação linear positiva apenas entre a largura do crânio com a largura do cerebelo $(\mathrm{P}$-valor $=0,029, \mathrm{r}=0,6233 \mathrm{e}$ $\left.R^{2}=0,3885\right)$.

Tabela 1. Valores da média e desvio padrão (em $\mathrm{cm}$ ) das medidas do crânio e do encéfalo de avestruzes, separadas por sexo.

STRUTHIO CAMELUS

$\begin{array}{llll}\text { MACHOS } & \text { FÊMEAS } & \text { P- } & \text { GERAL } \\ (\mathrm{N}=10) & (\mathrm{N}=10) & \text { VALOR } & (\mathrm{N}=20)\end{array}$

\begin{tabular}{l|llll}
\hline COMPRIMENTO DO CRÂNIO & $10,13 \pm 0,10$ & $9,99 \pm 0,15$ & $0,04^{*}$ & $10,06 \pm 0,14$ \\
LARGURA DO CRÂNIO & $9,31 \pm 0,11$ & $9,32 \pm 0,13$ & 0,88 & $9,32 \pm 0,12$ \\
COMPRIMENTO DO ENCÉFALO & $5,57 \pm 023$ & $5,56 \pm 0,23$ & 0,92 & $5,57 \pm 0,23$ \\
LARGURA DO ENCÉFALO & $4,19 \pm 0,16$ & $4,18 \pm 0,16$ & 0,89 & $4,18 \pm 0,16$ \\
COMPRIMENTO DO HEMISFÉRIO CEREBRAL & $3,41 \pm 0,13$ & $3,39 \pm 0,12$ & 0,74 & $3,41 \pm 0,13$ \\
DIREITO & & & & \\
COMPRIMENTO DO HEMISFÉRIO CEREBRAL & $3,35 \pm 0,14$ & $3,34 \pm 0,05$ & 0,86 & $3,35 \pm 0,12$ \\
ESQUERDO & & & &
\end{tabular}




\begin{tabular}{|c|c|c|c|c|c|c|c|}
\hline $\begin{array}{l}\text { LARGURA } \\
\text { DIREITO }\end{array}$ & DO & HEMISFÉRIO & CEREBRAL & $2,27 \pm 0,17$ & $2,25 \pm 0,14$ & 0,78 & $2,26 \pm 0,16$ \\
\hline $\begin{array}{l}\text { LARGURA } \\
\text { ESQUERDO }\end{array}$ & DO & HEMISFÉRIO & CEREBRAL & $2,25 \pm 0,11$ & $2,27 \pm 0,11$ & 0,71 & $2,26 \pm 0,12$ \\
\hline ALTURA DO F & HEM & SFÉRIO CEREBR & L DIREITO & $2,08 \pm 0,23$ & $2,07 \pm 0,21$ & 0,92 & $2,07 \pm 0,22$ \\
\hline \multicolumn{8}{|l|}{ ESQUERDO } \\
\hline COMPRIMEN' & NTO D & CEREBELO & & $2,45 \pm 0,32$ & $2,44 \pm 0,32$ & 0,95 & $2,44 \pm 0,32$ \\
\hline LARGURA DO & $\mathrm{O}$ CEI & EBELO & & $1,59 \pm 0,16$ & $1,58 \pm 0,15$ & 0,89 & $1,58 \pm 0,16$ \\
\hline COMPRIMEN' & NTO D & LOBO ÓPTICO & IREITO & $1,30 \pm 0,06$ & $1,30 \pm 0,06$ & 1,00 & $1,30 \pm 0,07$ \\
\hline COMPRIMEN' & NTO D & LOBO ÓPTICO & ISQUERDO & $1,24 \pm 0,07$ & $1,23 \pm 0,67$ & 0,75 & $1,24 \pm 0,07$ \\
\hline LARGURA DO & O LOI & O ÓPTICO DIREI & & $1,08 \pm 0,07$ & $1,08 \pm 0,07$ & 1,00 & $1,08 \pm 0,08$ \\
\hline LARGURA DO & $0 \mathrm{LO}$ & O ÓPTICO ESQU & RDO & $1,08 \pm 0,07$ & $1,08 \pm 0,07$ & 1,00 & $1,08 \pm 0,08$ \\
\hline${ }^{*} \mathrm{P}<0,05$ & & & & & & & \\
\hline
\end{tabular}

Tabela 2: Valores da média e desvio padrão $(\mathrm{em} \mathrm{cm})$ das medidas do encéfalo de avestruzes.

\begin{tabular}{l|lll}
\multicolumn{1}{l}{ Struthio camelus $(\mathbf{n}=\mathbf{2 0})$} & DIREITO & ESQUERDO & P-VALOR \\
\hline COMPRIMENTO DO HEMISFÉRIO CEREBRAL & $3,40 \pm 0,13$ & $3,40 \pm 0,12$ & 0,20 \\
LARGURA DO HEMISFÉRIO CEREBRAL & $2,30 \pm 0,16$ & $2,3 \pm 0,12$ & 0,91 \\
ALTURA DO HEMISFÉRIO CEREBRAL & $2,07 \pm 0,22$ & $2,05 \pm 0,23$ & 0,83 \\
COMPRIMENTO DO LOBO ÓPTICO & $1,30 \pm 0,07$ & $1,24 \pm 0,07$ & $0,01^{*}$ \\
LARGURA DO LOBO ÓPTICO & $1,08 \pm 0,08$ & $1,24 \pm 0,07$ & 1 \\
*P<0,05 & & &
\end{tabular}

\section{Morfologia geral do encéfalo}

\section{Vista dorsal}

Ambos os hemisférios cerebrais estão situados rostralmente, e o cerebelo e a medula espinhal estão situados caudalmente (Figura 2). Macroscopicamente não se observou diferenças na morfologia entre os hemisférios de machos e fêmeas. Observa-se uma fissura longitudinal entre os hemisférios cerebrais denominada de fissura inter-hemisférica; e um sulco transversal entre o cérebro e o cerebelo chamado de fissura subhemisférica (Figura 2). 
Os hemisférios cerebrais direito e esquerdo representam $61,22 \%$ e $60,14 \%$ respectivamente do comprimento total do encéfalo nos machos. Nas fêmeas esses valores são de 60,97\% para o hemisfério direito e 60,07\% para o hemisfério esquerdo.

A superfície cerebral é lisa e não possui giro ou sulco. No entanto, na superfície dorsal de cada hemisfério cerebral, uma depressão oblíqua denominada valécula telencefálica divide o hemisfério em uma parte rostromedial e outra caudolateral.

$\mathrm{O}$ cerebelo fica localizado caudal à fissura sub-hemisférica e dorsalmente à medula espinhal e está fixado ao bulbo, à ponte e ao mesencéfalo, pelos pedúnculos cerebelares caudal, intermediário (médio) e rostral respectivamente. Consiste de um corpo (vermis) localizado no centro do cerebelo com fissuras transversais, e dois hemisférios (aurículas) cerebelares situados em cada lado do corpo (Figura 2). Entretanto em uma vista lateral apresenta uma forma de losango.

Figura 2: Fotomacrografia do encéfalo de avestruz em vista dorsal. a: Hemisfério cerebral; b: valécula telencefálica; c: Fissura inter-hemisférica; d: lobo óptico; e; Fissura sub-hemisférica; f: cerebelo. Barra

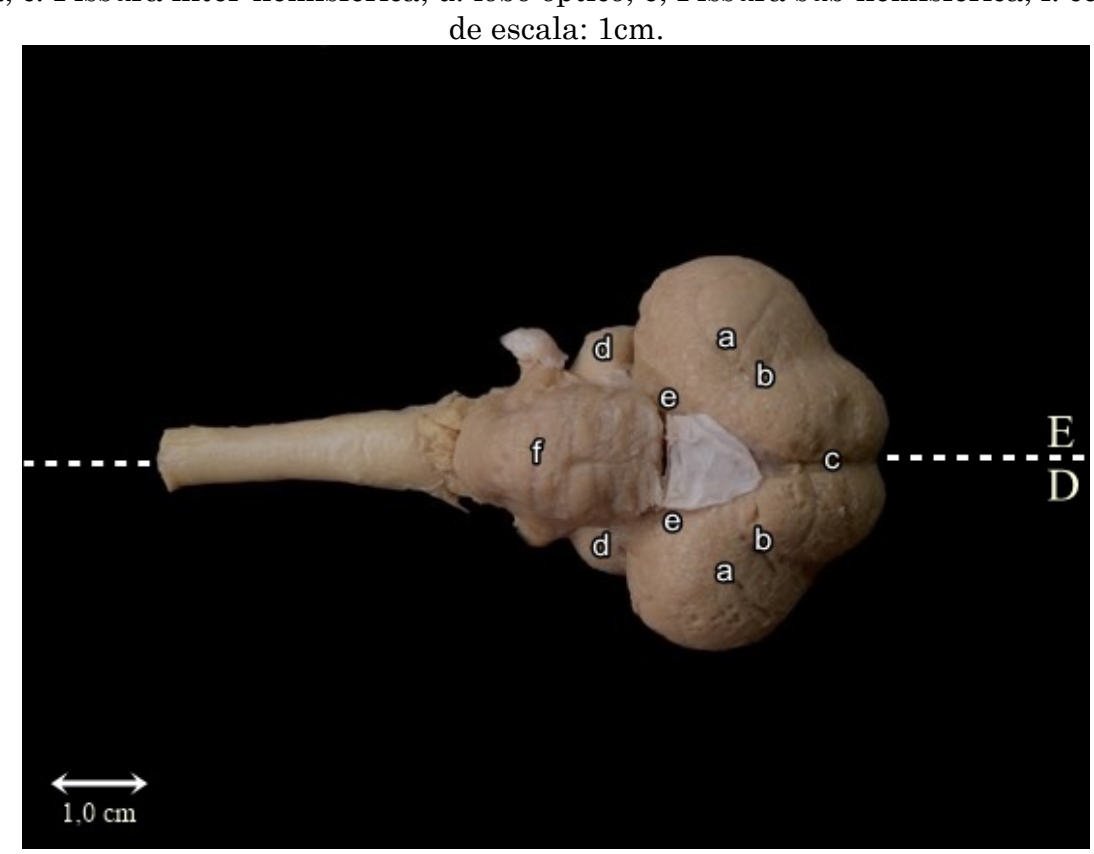




\section{Vista ventral}

Um pequeno bulbo olfatório (Figura 3) é observado na parte rostral de cada hemisfério cerebral. Não se observa trato olfatório. O quiasma óptico é central e aparece caudalmente à fissura mediana dos hemisférios cerebrais (Figura 3). O trato óptico do avestruz é desenvolvido. As fibras nervosas do trato óptico inclinam-se lateralmente e se cruzam, ligando-se ao lobo óptico do mesencéfalo. O lobo óptico é uma eminência oval localizada bilateralmente na parte ventral do mesencéfalo e converge na linha mediana ventral, recebendo as fibras do quiasma óptico. Ambos os lobos ópticos se projetam nas superfícies ventral e lateral do mesencéfalo. As estruturas localizadas caudalmente ao quiasma óptico pertencem ao tronco encefálico (mesencéfalo, ponte e bulbo).

No avestruz o tronco encefálico é constituído pelo mesencéfalo (situado rostralmente), pela ponte e pelo bulbo (situados caudalmente), inexistindo um limite macroscópico definido entre essas estruturas. A ponte não apresenta uma macroscopia nítida no cérebro de avestruz, estando conectada com o bulbo. A superfície do bulbo é lisa e em toda sua extensão observa-se a fissura mediana, que termina em uma depressão que dá origem ao mesencéfalo e forma os pedúnculos cerebrais. As pirâmides e a decussação das pirâmides não são evidentes (Figura 3). 
Figura 3: Fotomacrografia do encéfalo de avestruz em vista ventral. a: Hemisfério cerebral; b: bulbo olfatório; c: fissura mediana; d: quiasma óptico; e: lobo óptico. Barra de escala: $1 \mathrm{~cm}$.

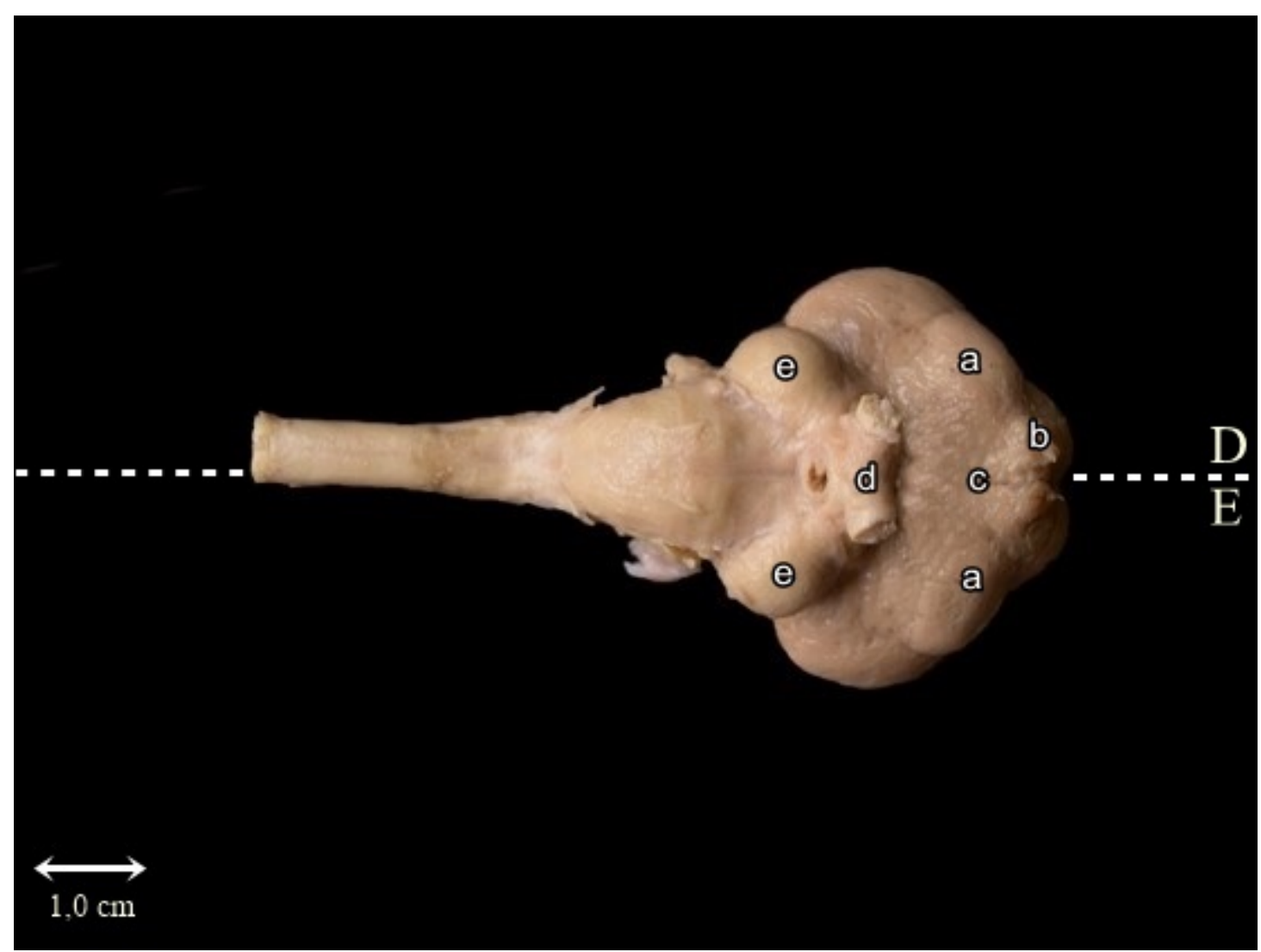

\section{Discussão}

O encéfalo das aves é dividido em três partes: prosencéfalo, mesencéfalo e rombencéfalo. É formado pelos hemisférios cerebrais direito e esquerdo, pela glândula pineal, pelos lobos ópticos e pelo cerebelo, quando vistos da superfície dorsal. Na superfície ventral, a partir da extremidade rostral à caudal, observam-se os bulbos olfatórios, as faces orbitais dos hemisférios cerebrais, o quiasma óptico, o trato óptico, os lobos ópticos, a hipófise, o mesencéfalo (pedúnculos cerebrais), a ponte e o bulbo que se continuam na medula espinhal, semelhante ao observado em Gallus domesticus (GUPTA, HASWITHA e KAUR, 2019), anseriformes (KALISINSKA, 2005), avestruz (PENG et al., 2010) e na presente investigação. 
Os hemisférios cerebrais direito e esquerdo estão situados na parte rostral do encéfalo. Observam-se também bulbos olfatórios pares e de dimensões reduzidas nas extremidades do cérebro, e a ausência de tratos olfatórios. Uma fissura inter-hemisférica é observada entre os hemisférios cerebrais direito e esquerdo (PENG et al., 2010). Essas informações corroboram os resultados obtidos na presente pesquisa; além disso não foram observadas diferenças estatísticas nas medidas dos hemisférios cerebrais quando foram comparados entre os sexos e os antimeros.

O cérebro do avestruz caracterizado neste experimento é do tipo lisencefálico, não havendo evidências de giros e sulcos, conforme relatado por Batah, Ghaje e Sh (2012) e Gupta, Haswitha e Kaur (2019) em frango. Essa ausência de giros e sulcos não parece ter relação com o número de neurônios (MOTA e HERCULANO-HOUZEL, 2015). No entanto, na superfície dorsal de cada hemisfério cerebral, uma depressão oblíqua denominada valécula telencefálica divide o hemisfério em uma parte rostromedial e outra caudolateral (PENG et al., 2010).

No avestruz, conforme evidenciado no presente estudo, em uma vista dorsal o hemisfério cerebral apresenta a forma de um triângulo obtuso, diferente da forma triangular observada no frango (BATAH, GHAJE e SH, 2012; GUPTA, HASWITHA e KAUR, 2019), da forma trapezoide observada em cegonha branca, e da forma de cones nos patos de Pequim e no ganso cinza (PENG et al., 2010).

O cerebelo no avestruz é desenvolvido e fica localizado caudal a fissura sub-hemisférica. Apresenta uma forma de losango em uma vista lateral. É mais comprido que largo semelhante ao observado por Batah, Ghaje e Sh (2012) e Gupta, Haswitha e Kaur (2019) em frangos. O cerebelo é separado do bulbo pelo quarto ventrículo, semelhante ao observado por Pal, Chowdhury e Ghosh (2003) e Gupta, Haswitha e Kaur (2019) em aves. A superfície dorsal média do cerebelo apresenta de giros transversais e sulcos. 
O cerebelo se conecta ao mesencéfalo rostralmente e ao bulbo caudalmente (KÖNIG, KOLBEL e LIEBICH, 2016).

\title{
Conclusão
}

O cérebro do avestruz é classificado como lisencefálico não apresentando giros e sulcos. O comprimento dos crânios dos machos é maior que o das fêmeas. Em relação aos 20 encéfalos analisados o lobo óptico direito é maior que o esquerdo. Houve correlação linear positiva entre a largura do crânio com a largura do cerebelo.

\section{Agradecimentos}

Fundação de Amparo à Pesquisa do Estado do Rio de Janeiro (FAPERJ), Conselho Nacional de Desenvolvimento Científico e Tecnológico (CNPq) e Coordenação de Aperfeiçoamento de Pessoal de Nível Superior (Capes) - Código de Financiamento 001.

\section{Morphology and morphometry of the ostrich encephalus (Struthio camelus)}

\begin{abstract}
The african ostrich is a ratite bird in the family Struthionidae. The anatomical features of the African ostrich brain were investigated in this study. Twenty adult ostrich heads (10 males and 10 females), fixed in $10 \%$ formaldehyde, were analyzed in this study. The measures of the brain were obtained: length and total width of the brain, length, width and height of the right and left cerebral hemispheres, length and width of the cerebellum, length and width of the right and left optical lobes. The length and width of the skull were also measured. Results for each variable were expressed as mean and standard deviation. Through unpaired Student's ttest the variables were compared between sexes and between antimers. Statistical significance was considered as $\mathrm{p}<0.05$. The length and width of the male encephalus was $5.57 \pm 0.23 \mathrm{~cm}$ and $4.19 \pm 0.16$, respectively. In females, the length and width of the encephalus were $5.56 \pm 0.23$ and 4.18 \pm 0.16 respectively. The length of the right cerebral hemisphere was $3.41 \pm$ 0.13 in males and $3.39 \pm 0.12$ in females, while in the left it was $3.35 \pm 0.14$
\end{abstract}


in males and $3.34 \pm 0.05$ in females. The width of the right cerebral hemisphere was $2.27 \pm 0.17$ in males and $2.25 \pm 0.14$ in females, and the left was $2.25 \pm 0.11$ in males and $2.27 \pm 0.11$ in females. The height of the right cerebral hemisphere was $2.08 \pm 0.23$ in males and $2.07 \pm 0.21$ in females and the left cerebral hemisphere was $2.05 \pm 0.22$ in males and 2.05 \pm 0.22 in females. The length of the cerebellum was $2.45 \pm 0.32$ in males and $2.44 \pm 0.32$ in females, and its width was $1.59 \pm 0.16$ in males and 1.58 \pm 0.15 in females. The length of the right optic lobe was $1.30 \pm 0.06$ in males and $1.30 \pm 0.06$ in females, while the length of the left optic lobe was $1.24 \pm 0.07$ in males and $1.23 \pm 0.67$ in females. The width of the right optic lobe was $1.08 \pm 0.07$ in males and $1.08 \pm 0.07$ in females and the width of the left optic lobe was $1.08 \pm 0.07$ in males and $1.08 \pm 0.07$ in females. The surface of the ostrich brain is smooth without any gyrus or sulcus. The average length of the skulls of males is greater than that of females. In relation to the 20 encephalus analyzed, the right optic lobe is larger than the left. There was a positive linear correlation between the width of the skull and the width of the cerebellum.

Keywords: Anatomy, Birds, Ratites, Nervous system.

\section{Referências}

ASPRILLA-PEREA, J.; DÍAZ-PUENTE, J. M.; Importance of wild foods to household food security in tropical forest areas. Food Security, St Paul, v. 11, p. 1522, October 2019. https://doi.org/10.1007/s12571-018-0846-8

BATAH, A. L.; GHAJE, M. S.; SH, N. A. Anatomical and histological study for the brain of the locally breed chicken (Gallus domesticus). Journal Thi-Qar Science, Baghdad, v. 3, n. 3, p. 47-53, August 2012.

GEUTHNER, A. C.; KOETHE, M.; LUDEWIG, M.; POTT, S.; SCHARES, G.; MAKSIMOV, P.; DAUGSCHIES, A., BANGOURA, B. Development of an in vivo model for Toxoplasma gondii infections in chickens and turkeys simulating natural routes of infection. Veterinary Parasitology, Amsterdam, v. 276, December 2019. https://doi.org/10.1016/j.vetpar.2019.108956

GUPTA, S. K.; HASWITHA, S.; KAUR, A. J. Gross Morphometrical Study on the Brain of Fowl (Gallus domesticus). The Indian Journal of Veterinary Sciences and 
Biotechnology, New Delhi, v.14, n. 4, p. 32-35, April 2019. https://doi.org/10.21887/ijvsbt.14.4.9

KALISIŃSKA E. Anseriform brain and its parts versus taxonomic and ecological categories. Brain, Behavior and Evolution, New York, v. 65, n. 4, p. 244-261, March 2005. https://doi.org/10.1159/000084315

KAWABE, S.; SHIMOKAWA, T.; MIKI, H.; MATSUDA, S.; ENDO, H. Variation in avian brain shape: relationship with size and orbital shape. Journal of Anatomy, London, v. 223, n. 5, p. 495-508, September 2013. https://doi.org/10.1111/joa.12109

KÖNIG, H. E.; KORBEL, R.; LIEBICH, H. G. Avian Anatomy: Textbook and Colour Atlas. 2. ed. Sheffield: 5M Publishing Ltd, 2016. 358p.

KOTRSCHAL, A.; CORRAL-LOPEZ, A.; AMCOFF, M.; KOLM, N. A larger brain confers a benefit in a spatial mate search learning task in male guppies. Behavioral Ecology, New York, v. 26, n. 2, p. 527-532, March-April 2015. https://doi.org/10.1093/beheco/aru227

LÜPS, P. 'Haller's law' (1762): the observation of a relation between brain weight and body weight by Albrecht von Haller (1708-1777). Mammalian Biology, Berlin, v. 75, n. 6, p. 577-579, November 2010. https://doi.org/10.1016/j.mambio.2009.11.005

MOTA, B.; HERCULANO-HOUZEL, S. Cortical folding scales universally with surface area and thickness, not number of neurons. Science, New York, v. 349, n. 6243, p. 74-77, July 2015. https://doi.org/10.1126/science.aaa9101

PAL, B.; CHOWDHURY, S.; GHOSH, R. K. Comparative anatomical study of the cerebellum of man and fowl. Journal of anatomical society of India, Raipur, v. 52, n. 1, p. 32-37, January-March 2003. 
PENG, K.; FENG, Y.; ZHANG, G.; LIU, H.; SONG, H. Anatomical study of the brain of the African ostrich. Turkish Journal of Veterinary and Animal Sciences, Ankara, v. 34, n. 3, p. 235-241, June 2010. https://doi.org/10.3906/vet-0806-19

RICHTSMEIER, J. T.; FLAHERTY, K. Hand in glove: brain and skull development and dysmorphogenesis. Acta Neuropathologica, Berlin, v. 125, n. 4, p. 469-489, April 2013. https://doi.org/10.1007/s00401-013-1104-y

STRIEDTER, G. F. History of ideas on brain evolution. In: KAAS, J. H. Evolutionary Neuroscience. Oxford: Academic Press, 2009, cap. 1, p. 3-18.

YU, E.; ASHWELL, K. W. S.; SHULRUF, B. Quantitative analysis of arterial supply to the developing brain in tetrapod vertebrates. The Anatomical Record, Hoboken, November 2019. https://doi.org/10.1002/ar.24317. [Epub ahead of print] 